\title{
NEBULAR SPECTRUM OF PU VUL IN 2004
}

\author{
Kye-Hwa Yoo \\ Department of Science Education, Ewha Womans University, Seoul, 120-750, Korea \\ E-mail:khyoo@ewha.ac.kr \\ (Received January 19, 2007; Accepted May 16, 2007)
}

\begin{abstract}
A high resolution spectrum of PU Vul obtained at Bohyunsan Astronomy Observatory on April 9, 2004 is presented. At this phase, PU Vul was an emission-line star and its continuum was very weak. Emission lines of He II, H I, [Ne IV], [N II], [O III], [Ar V ] and [Fe VII] dominated the spectrum of PU Vul. Many of them exhibited hat-top profiles with strong and multi-peaked emissions on flat-tops of their profiles. Radial velocities for these lines were measured. Origins of the spectral lines are discussed in terms of the wind and the photoionization models.
\end{abstract}

Key words : symbiotic star: PU Vul — high resolution spectrum : line variations — geometrical structure

\section{INTRODUCTION}

PU Vul was an irregular variable M6.5 III that showed small light variations of $\mathrm{m}=15-16$ in its $\mathrm{B}$ light curve before the outburst (Liller \& Liller, 1979). The outburst of PU Vul started in November 1977 and PU Vul gradually brightened after that time (Kuwano, 1979; Honda et al., 1979; Yamashita, 1979).

In March 1979, its brightness was at $m=9.3$ and it remained in the state of constant maximum brightness for about 8.5 years except for an abrupt decrease in 1980 and a gradual brightening a year latter. Then its brightness begun to gradually decrease from September 1987 (Yamashita et al., 1982, 1983; Maitzen et al., 1987) and was about $\mathrm{m}=12.5$ in 2002 (Netopil, 2002). Since the eruption, PU Vul experienced two eclipse phases; 1980-1982 and 1993-1994 (Yamashita et al., 1982; Iijima, 1989; Belyakina et al., 1989; Kanamitsu, 1991; Nussbaumer \& Vogel, 1996).

At its maximum brightness, PU Vul showed the spectrum of a supergiant of A- or F-type (Yamashita et al., 1982; Yamashita et al., 1983). From 1987, it began to show a nebular spectrum. Compared with a nova, the brightness of PU Vul increased more slowly toward the maximum and its light curve showed the plateau for a few years. Its spectrum had symbiotic characteristics in the recent years. Hence it was referred as a slow nova-like symbiotic star.

In order to examine the photometric and spectroscopic characteristics of this symbiotic nova, PU Vul, a large amount of data was collected, at visible spectroscopic wavelengths (Yamashita et al., 1982, 1983; Iijima \& Ortolani, 1984; Kenyon, 1986; Iijima, 1989; Kanamitsu et al., 1991; Kanamitsu, 1991; Tomov et al., 1991; Gochermann, 1991; Tamura et al., 1992; Klein et al., 1994; Andrillat \& Houziaux, 1994, 1995), in the optical photometry (Nakagiri \& Yamashita, 1980; Belyakina et al., 1982, 1984, 1989; Kolotilov, 1989; Kolotilov et al., 1995), in UV and X-ray wavelengths (Vogel \&
Nussbaumer, 1992 ; Nussbaumer \& Vogel, 1996; Hoard et al., 1996) and in IR wavelengths (Bensammar et al., 1991).

A binary model was proposed at the first and second eclipsing times (Belyakina et al., 1989; Iijima, 1989; Bensammar et al., 1991; Kanamitsu et al., 1991). Subsequently, a triple model was proposed based on the radial velocity of the hot white dwarf (Chochol et al., 1997), which consists of the inner binary of both a white dwarf and a bright $\mathrm{M}$ giant with an orbital period of 760-day and the outer object of a cool M giant with that of 4900-day (Chochol et al., 1997). On the other hand, the cool $\mathrm{M}$ giant has a pulsation period of about 217-day, which was determined by photoelectric UBVR observations (Chochol et al., 1997, 1998).

In this paper, we report the spectrum of PU Vul obtained in April 2004. In the second Section, an observation and data reduction processes are presented. The spectral behavior of PU Vul obtained in April 2004 is reported in the third Section. Gaussian de-convolutions for $\mathrm{H} \alpha, \mathrm{He}$, $[\mathrm{O} \mathrm{III}]$ and [Fe VI] are described in the fourth Section. Measured radial velocities for all observed lines are presented in the fifth Section. Finally the origin of observed lines and a geometric structure of PU Vul are discussed.

\section{OBSERVATIONS}

A spectroscopic observation was carried out by using the echelle spectrograph mounted on $1.8 \mathrm{~m}$ telescope at the Bohyunsan Astronomical Observatory. In April 2004, PU Vul had a visual magnitude of about 12 (AAVSO). During the observing term in April 2004, PU Vul was not a main target but rather we wanted to observe on occasions. However, due to bad weather conditions only one spectrum for PU Vul was obtained on April 9(UT), 2004.

A CCD detector of $2024 \times 4096$ pixels with a size of $15 \times 15 \mu \mathrm{m}$ per pixel, the fiber's diameter of 200 


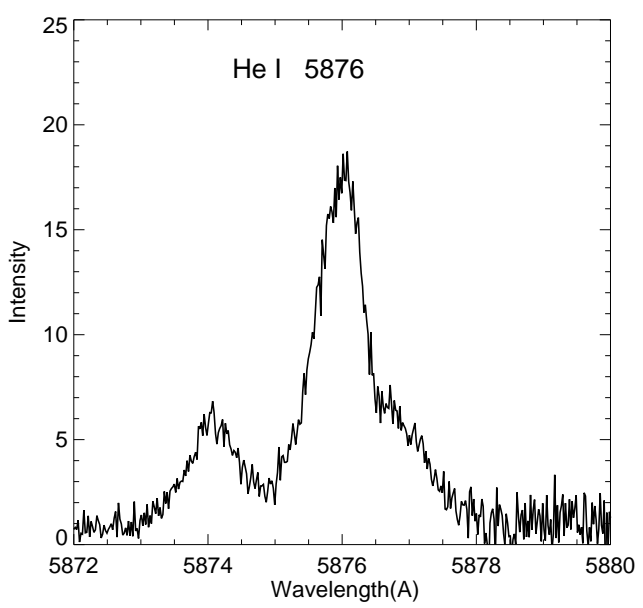

Fig. 1. - The weak emission line profile of He I $\lambda 5876$.

$\mu \mathrm{m}$ and a 41 -groove $\mathrm{mm}^{-1}$ grating were used. Exposure time was 1200 seconds. BOES reaches $\mathrm{S} / \mathrm{N}$ (signal to noise $)=25$ for a 11th magnitude star in 30 minutes during the seeing of 2.5" (Kim, 2004). The obtained resolving power was $\lambda / \Delta \lambda=45000$ for the $200 \mu \mathrm{m}$ fiber. The achieved spectrum ranged from $\lambda 3700$ to $\lambda 9000$ and its output dispersion was covered from 1.56 to $2.14 \AA \mathrm{mm}^{-1}$. Using Th-Ar and halogen lamps, we calibrated wavelengths and a flat fielding, respectively. The standard data reduction procedures were performed using IRAF tools. The obtained spectrum was normalized to the local continuum.

\section{SPECTRAL VARIATIONS}

Since 1997 the spectra of PU Vul have not been reported over a long term. On April 9, 2004 there might be some appreciable variations in spectral lines of $\mathrm{PU}$ Vul.

Line identification of the spectrum observed in April 2004 was done using line lists of RR Tel (Thackeray \& Webster, 1974), V1016 Cyg (Fitzgerald \& Pilavaki, 1974 ; Andrillat et al., 1982) and the revised mutiplet table of elements (Moore, 1958). The line list was given by Yoo (2005) in a preliminary report of PU Vul.

He I $\lambda 4471$ began to show a weak emission in 1987 (Iijima, 1989) and showed a single-peaked strong emission from 1990 (Tomov et al., 1991) to 1994 (Andrillat \& Houziaux, 1995). In April 2004, He I $\lambda 5876$ had a complex profile that split into double-peaked components with the strongest redward emission.

He I line was originated in the inner part of the accretion disk with a double-peaked profile. Figure 1 gives the line profile of He I $\lambda$ 5876. Similarity to the $\mathrm{H} \alpha$ profile was noted.

He II $\lambda 4686$ and $\lambda 5412$ appeared as a broad emission in around 1990 (Tomov et al., 1991) with a doublepeaked emission (Andrillat \& Houziaux, 1995) and ex-

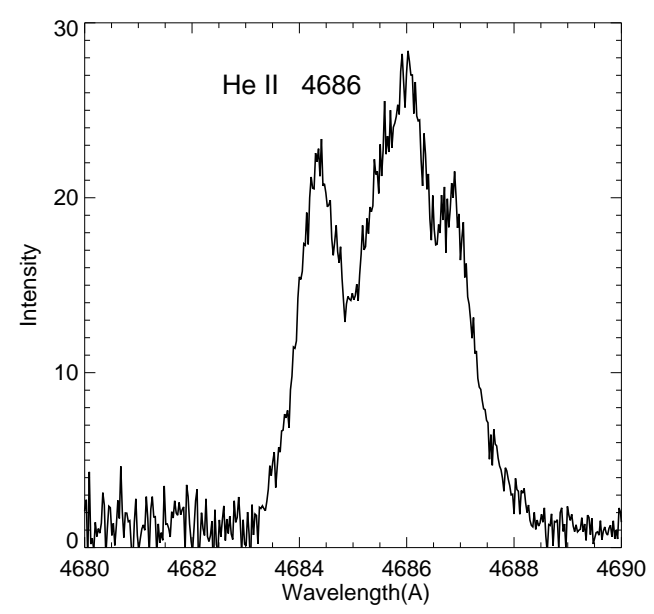

Fig. 2. - The line profile of He II $\lambda 4686$.

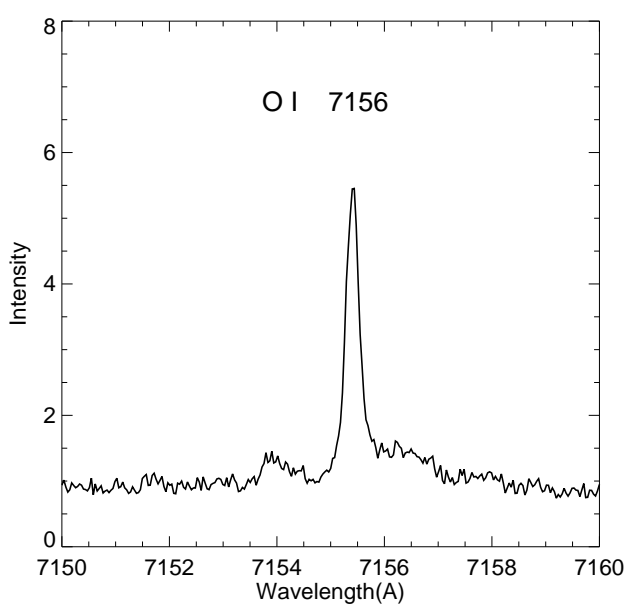

Fig. 3. - The line profile of O I $\lambda 7156$.

hibited as a very broad emission in November 1994, when PU Vul was at the end of the second eclipse (Kolotilov et al., 1995). This indicates that the size of the region forming He II lines might be comparably larger than expected. The line of He II $\lambda 4686$ is shown in Figure 2.

In April 2004, O I $\lambda 7157$ had a broad emission feature with the blue and red components and a strong central emission component positioned asymmetrically around the center of the line. The intensity of the blue component was stronger than the red component. The line features of $\mathrm{O}$ I and He I were very similar but the intensity of O I $\lambda 7157$ line was weaker by about a fifth part than that of He I $\lambda 5876$ line. The line profile of O I $\lambda 7157$ is shown in Figure 3.

From 1992-1994, highly ionized elements of [N II], [Ar IV] and [Fe IV] that were formed by collisions were observed but their intensities were not weak (Andrillat 


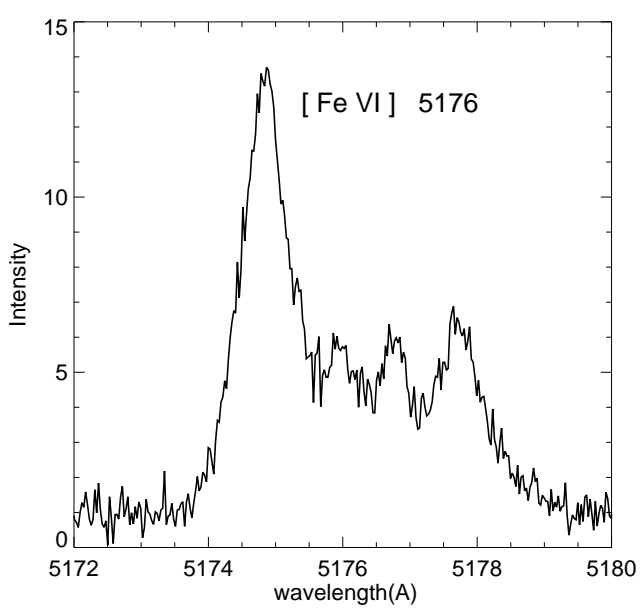

Fig. 4. - The line profile of $[\mathrm{Fe} \mathrm{VI}] \lambda 5176$.

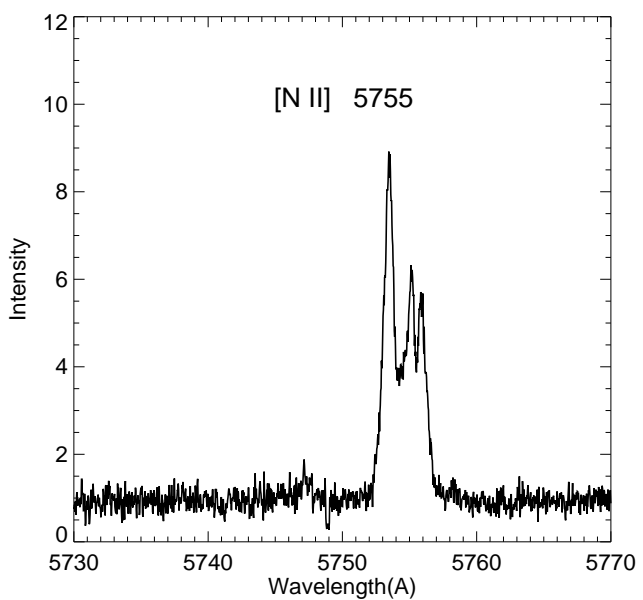

Fig. 5. - The line profile of $[\mathrm{N} \mathrm{II}] \lambda 5755$.

\& Houziaux, 1995; Kolotilov et al., 1995). However, in April 2004, [N II]. [Ar V] and [Ca V] were appeared weak, and $[\mathrm{Fe} \mathrm{VI}]$ and $[\mathrm{Fe} \mathrm{VII}]$ were appeared strong. The intensities of [Fe VI] and [Fe VII] were the highest except for H I, He I, [O I] and [O III]. Figure 4 presents $[\mathrm{Fe}$ VI] line around $\lambda$ 5176. Line profiles of $[\mathrm{N} \mathrm{II}] \lambda 5755$, $[\mathrm{Ar} \mathrm{V}] \lambda 6435,[\mathrm{O} \mathrm{I}] \lambda 6300$ and $[\mathrm{Ca} \mathrm{V}] \lambda 5309$ are also displayed in Figures 5, 6, 7 and 8, respectively. [Ne IV] $\lambda 4725$ and [Ar III] $\lambda 7136$ which are to be discussed in Section 6 are displayed in Figures 9 and 10.

Since the 1979 outburst, the time sequential variations of $\mathrm{H} \mathrm{I}$ were as follow. The absorption of $\mathrm{H}$ I was weakened from April 1979 and disappeared in April 1987 (Yamashita et al., 1982, 1983; Iijima, 1989). Thereafter H I changed into the P-Cygni profile and further into various line shapes of single-peaked emissions, double-peaked emissions with wide wings and triple and quadruple-peaked emission lines (Kanamitsu

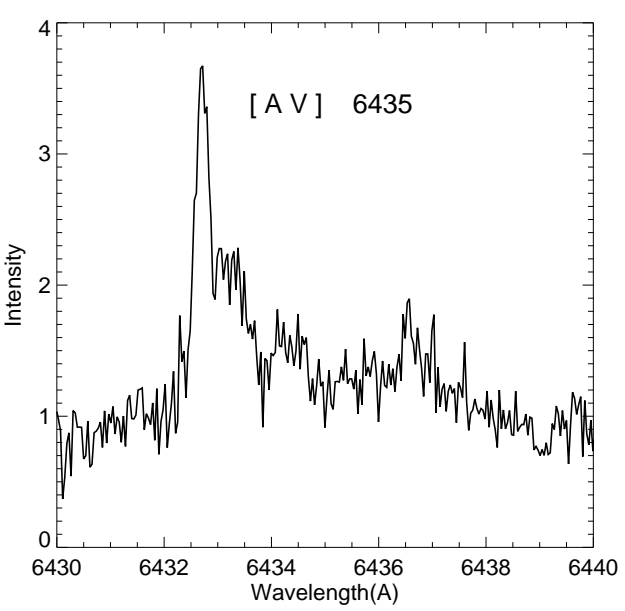

Fig. 6. - The line profile of $[\mathrm{Ar} \mathrm{V}] \lambda 6435$ and the sharp emission of $\lambda 6433$ Fe II. The sharp $\lambda 6433$ Fe II was observed on the blue wavelength wing of $\lambda 6435[\mathrm{Ar} \mathrm{V}]$ line.

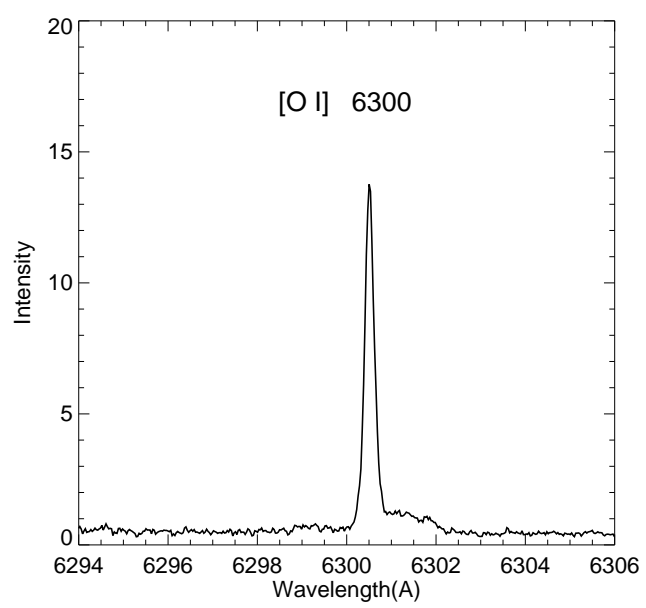

Fig. 7.- The line profile of $[\mathrm{O}$ I $] \lambda 6300$.

et al., 1991). The emission of $\mathrm{H} \beta$ appeared in September of 1983 and the emission of $\mathrm{H} \epsilon$ followed in December 1987 (Kanamitsu et al., 1991). In April 2004, H I was observed with at least two components, while in October 1987, it was observed as a single component. $\mathrm{H}$ I might have originated in the accretion disk. The slow wind due to the thermonuclear runaway from the white dwarf might provide evidence of sequential time variations. Figure 11 shows the intensity feature of the $\mathrm{H} \alpha$ in April 2004.

Weak [Ne III] and [O III] emerged in 1990 (Tomov et al., 1991). [O III] lines were enhanced in 1991 and had broad profiles in October 1994 (Kolotilov et al., 1995). In April 2004, [O III] lines showed triple-peaked component structure responsible for cloudlet material. The line of [O III] $\lambda 5007$ is displayed in Figure 12. In 


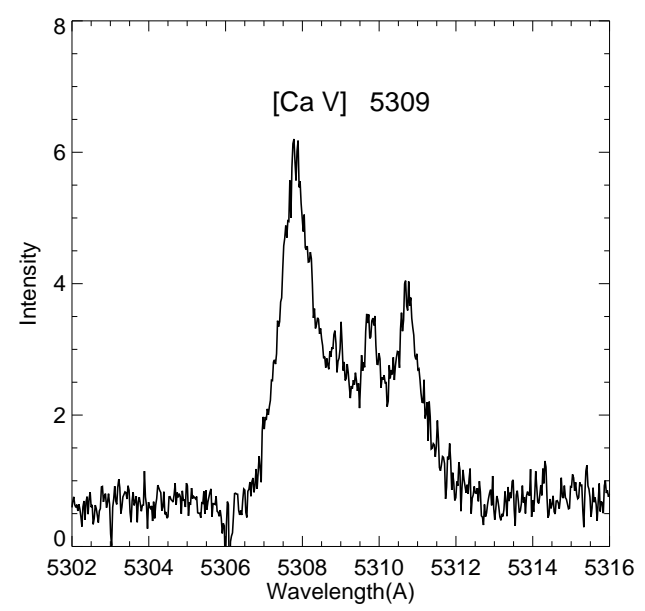

Fig. 8. - The line profile of $[\mathrm{Ca} \mathrm{V}] \lambda 5309$.

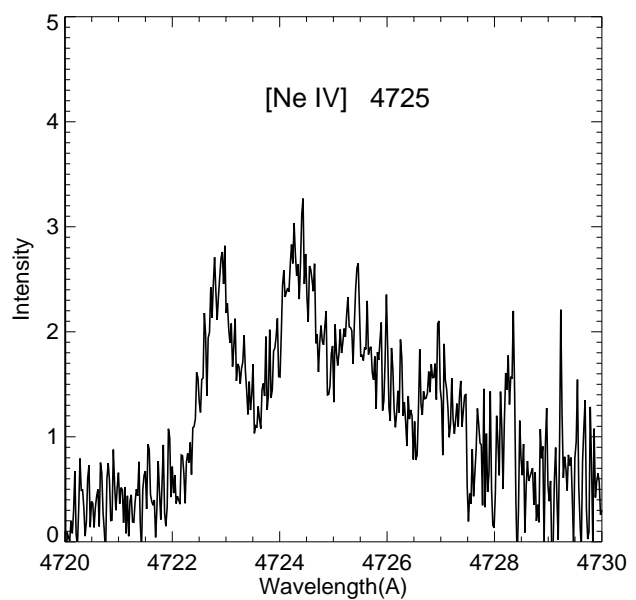

Fig. 9. - The line profile of $[\mathrm{Ne}$ IV] $\lambda 4725$.

April 2004, all relatively low ionized elements of [O II] and [Fe III] were not observed completely.

In 1988, many emissions of Fe II and [Fe II] appeared strong (Kanamitsu et al., 1991). However, they were gradually weakened thereafter. In April 2004, some of Fe II lines were observed with four emission components rather than a single component(see Figure 6).

In April 2004, the reason for the intensity being lower than the continuum level in the wide wavelength range of around $\lambda 7055$ might be the presence of the trace of the $(0,0)$ band head of $\gamma$-system of TiO.

\section{GAUSSIAN FITTING}

In Section 3, we described spectral variations of spectral lines of PU Vul in detail. All lines showed hattop line profiles with a few emission components superposed on their flat tops.

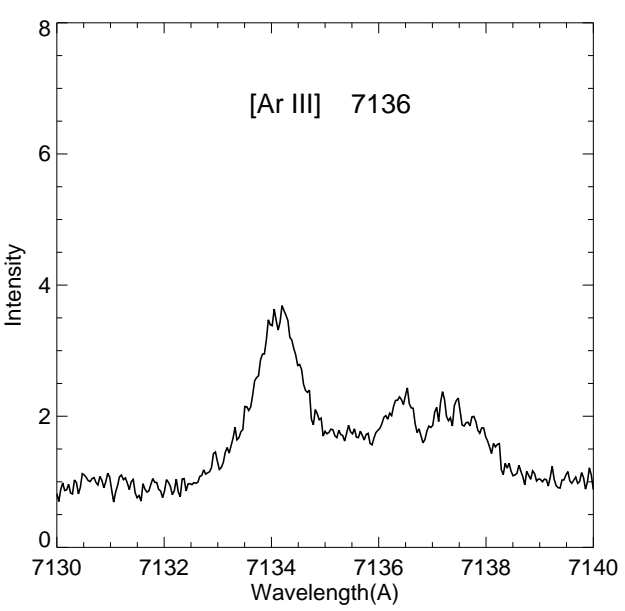

Fig. 10. - The line profile of $[$ Ar III $] \lambda 7136$.

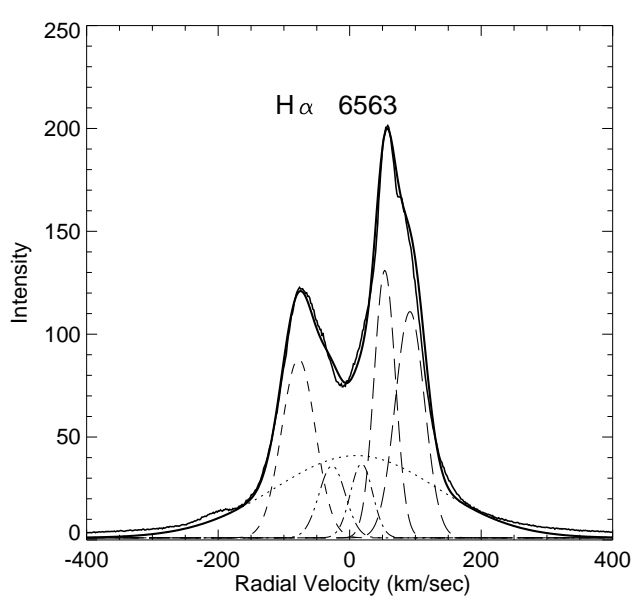

Fig. 11. - Gaussian deconvolution for the double-peaked line profile of $\mathrm{H} \alpha$.. The solid line gives the observed line. The bold solid, the dotted, the dashed and the dash dotted lines present the fitting result and Gaussian components.

To find out how the emission components of such line complexes were formed, we de-convolute the observed emission lines with Gaussian functions. Velocity fields of matter in a region originated the emission components is given by

$$
I\left(v, v_{0}, A, \sigma\right)=\Sigma A \exp \left(-\omega^{2}\right),
$$

where $\omega=\left(v-v_{0}\right) / \sigma, \sigma$ is the standard derivation, $v_{0}$ is the velocity at the center of Gaussian function, and $\mathrm{A}$ is a normalization factor.

Full width at half maximum (FWHM) of the Gaussian function is given by $\mathrm{FWHM}=2.355 \sigma$ (Ikeda \& Tamura, 2004). A program used to de-convolute the observed line into Gaussian functions was written by Dr. J. Chae. 


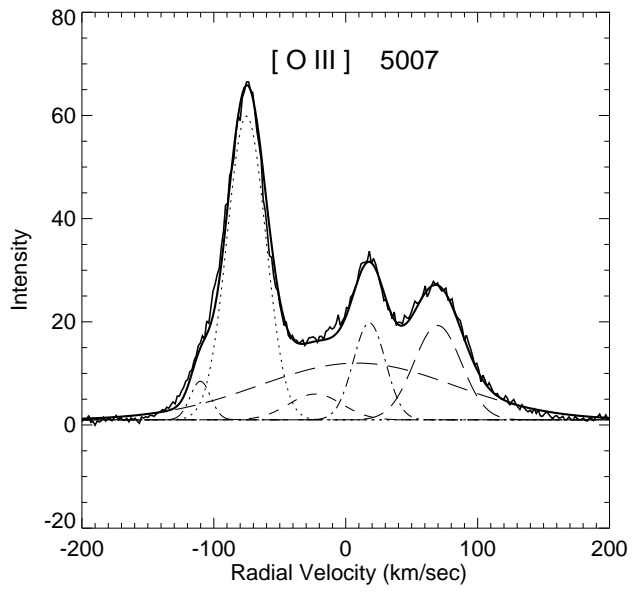

Fig. 12.- Gaussian deconvolution for the $\lambda 5007$ [O III]. The solid line gives the observed line. The bold solid, the dotted, the dashed and the dash dotted lines present the fitting result and Gaussian components.

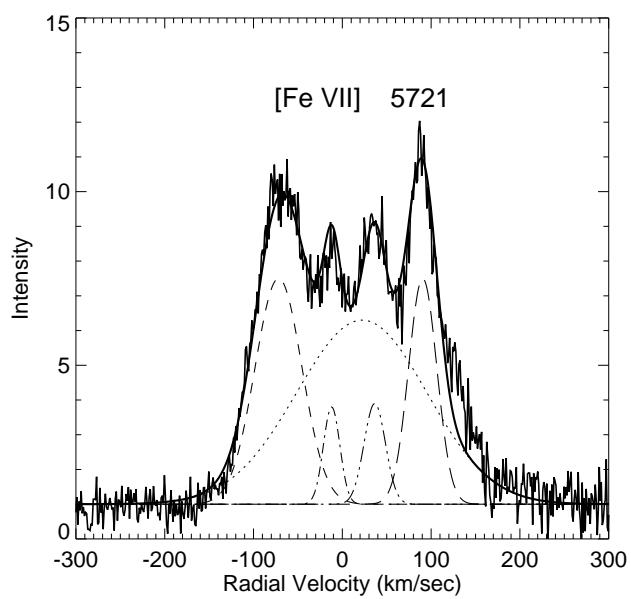

Fig. 13. - The line profile of $[\mathrm{Fe}$ VII $] \lambda$ 5721. The solid line gives the observed line. The bold solid, the dotted, the dashed and the dash dotted lines present the fitting result and Gaussian components.

The parameters of the Gaussian function are aggregated in Tables 1 and 2, where $\lambda_{0}$ is the wavelength at the center of the de-convoluted Gaussian function and $\mathrm{I}$ is the intensity of the Gaussian function from a local continuum. $V_{r}$ and $V_{D}$ are the radial velocity and Doppler velocity of each Gaussian component.

Each emission profile was de-convoluted into a central broad component (hereafter designated as B) and several narrow components. The latters are numbered according as wavelengths.

Table 1 is the de-convoluted result for $\mathrm{H} \alpha$, He I and He II. The de-convoluted resulting line profile for $\mathrm{H} \alpha$ is shown in Figure 11.
The velocity at the center of the broad B component is about $4 \mathrm{~km} \mathrm{~s}^{-1}$ close to the systemic velocity of about $6 \mathrm{~km} \mathrm{~s}^{-1}$ (see Figure 19 in Belyakina et al. 1989). The expanding velocity of matter that formed the B component was approximately a few hundred $\mathrm{km} \mathrm{s}^{-1}$. The emission component of H I first appeared in 1989, disappeared and reappeared after that time (Yamashita et al., 1982, 1983; Kanamitsu et al., 1991; Tamura et al., 1992; Klein et al., 1994). Therefore, the existence of the accretion disk that produced the Balmer line profiles might have happened around the end of the 1980s.

The He II region distant from the hot star might be ionized by a collision between the expanding He II cloud and the interstellar matter, in which then recombination takes place. If the He I line was yielded from the accretion disk, they might be formed in the inner region of the disk.

Forbidden lines of [O III], [Fe IV] and [Fe V] had hat-top profiles as mentioned in Section 3. They are known to be formed in a less dense region of spherically expanding envelope. The results of the Gaussian de-convolution are included together in Table 2. Each of the line profiles of [O III], [Fe IV] and [Fe V] had three or four emission components on the flat-top profiles. They had different radial velocities and may thus originate from different parts in the structure of expanding envelope. The de-convoluted line profiles for [O III] $\lambda 5007$ and [Fe VII] $\lambda 5721$ are shown in Figures 12 and 13 .

\section{RADIAL VELOCITIES}

In April 2004, PU Vul was relatively faint. The exposure time of 1200 seconds was insufficient to determine the center of each of components. Hence, there might be uncertainties regarding the radial velocities which were more than $1 \mathrm{~km} \mathrm{~s}^{-1}$.

The radial velocities of emission lines of $\mathrm{H} \mathrm{I},[\mathrm{O} \mathrm{I}]$, [O III]. [Ne IV], [Ca V], Fe II, [Fe II], [Fe VI] and [Fe VII] were measured. All velocities were corrected to the Sun. The radial velocities of a few main and probably available emission components of elements given at the bottom of Tables 3 and 4 were measured. No probable error in measured radial velocities means that it was a case of only one line. The results are given in Tables 3 and 4 .

When we measured radial velocities, the center at the height of about $80-90 \%$ of line intensities from the continuum was used. However, line-to-line radial velocity differences were conspicuously large.

Somewhat different radial velocities of Balmer line emission components around the line centers between blueward and redward peaks were observed. This means that the velocity field structures of the shell-like regions on the line of sight were somewhat complicated. The velocity of $[\mathrm{O} \mathrm{I}]$ might present the system velocity. Since the mean velocities of Fe II and [Fe II] were about $10 \mathrm{~km} \mathrm{~s}^{-1}$, they were also considered to have 
TABLE 1.

Parameters of the Gaussian components for H $\alpha$, He I and He II.

\begin{tabular}{|c|c|c|c|c|c|c|c|}
\hline $\begin{array}{l}\text { Date } \\
2004\end{array}$ & $\begin{array}{c}\text { Element } \\
\text { wavelength }(\AA)\end{array}$ & Component & $\overline{\lambda_{0}}$ & $\overline{\mathrm{I}}$ & $\begin{array}{c}\text { FWHM } \\
(\AA)\end{array}$ & $\begin{array}{r}V_{r} \\
\left(\mathrm{~km} \mathrm{~s}^{-1}\right)\end{array}$ & $\begin{array}{r}V_{D} \\
\left(\mathrm{~km} \mathrm{~s}^{-1}\right)\end{array}$ \\
\hline \multirow[t]{6}{*}{ April 9} & & (1) & 6560.92 & 87.00 & 1.31 & -77.7 & 72.2 \\
\hline & $\mathrm{H} \alpha$ & $(2)$ & 6562.03 & 35.00 & 1.03 & -26.6 & 56.7 \\
\hline & 6562.82 & $\mathrm{~B}$ & 6562.84 & 45.00 & 6.36 & +10.4 & 350.3 \\
\hline & & $(3)$ & 6563.01 & 36.00 & 0.88 & +18.1 & 48.5 \\
\hline & & (4) & 6563.78 & 130.00 & 0.84 & +53.4 & 46.3 \\
\hline & & (5) & 6564.62 & 110.00 & 1.15 & +91.7 & 63.3 \\
\hline \multirow[t]{5}{*}{ April 9} & & (1) & 5874.09 & 4.60 & 0.82 & -84.8 & 50.5 \\
\hline & He I & $(2)$ & 5875.25 & 1.50 & 0.51 & -25.9 & 31.4 \\
\hline & 5875.75 & $\mathrm{~B}$ & 5875.89 & 0.85 & 4.09 & +7.1 & 251.6 \\
\hline & & $(3)$ & 5875.99 & 16.00 & 0.80 & +12.4 & 49.2 \\
\hline & & (4) & 5876.92 & 3.70 & 0.74 & $\begin{array}{r}59.7 \\
\end{array}$ & 45.5 \\
\hline \multirow[t]{6}{*}{ April 9} & & (1) & 4684.22 & 11.70 & 0.58 & -84.3 & 44.7 \\
\hline & He II & $(2)$ & 4684.55 & 8.00 & 0.55 & -63.2 & 42.4 \\
\hline & 4685.68 & (3) & 4685.34 & 7.80 & 0.50 & -12.4 & 38.6 \\
\hline & & B & 4685.86 & 10.00 & 2.60 & +20.8 & 200.6 \\
\hline & & (4) & 4686.02 & 16.50 & 0.72 & +31.2 & 55.5 \\
\hline & & $(5)$ & 4686.92 & 11.70 & 0.55 & +88.6 & 42.4 \\
\hline
\end{tabular}

B: Gaussian broad emission component.

$(1),(2) \ldots(5)$ : Gaussian emission components in the order of wavelength.

originated close to the hot white dwarf. Hence it might be the approximate system velocity in April 2004.

\section{DISCUSSION AND CONCLUSION}

We introduced the accretion disk and the wind-shock and photoionization models for interpreting the characteristics of each line identified in April 2004.

The wind was attributed to the thermonuclear runaway of the accreted material from the surface of the white dwarf, which was considered to have moved outward symmetrically. PU Vul is known to have experienced the outburst by CN cycle(Iben \& Tutukov, 1996). A few Gaussian density profiles and linear velocity profiles were predicted as a function of the distance from the white dwarf. The linear relationship between line intensities and expansion velocities of Gaussian components might be anticipated for these values in Table 2 . The accretion disk had its own characteristic density and velocity profiles.

Matter transferred to the white dwarf during the periastron passage of the third object is increased (Chochol et al. 2003). If mass accretion was larger than a critical value, the other intermediate outward wind could be taken placed along the rotational axes of the white dwarf. The head-on collision between the outward wind from the white dwarf and the material distributed in the circumstellar space far from the white dwarf produced a shock front in their respective velocity fields. The redistribution of the circumstellar material as a result of the shock caused it to form some bloblike cloudlets at the peaks of the shells. The resulting density and temperature might be relatively higher, following the above mentioned distribution curves.

H I appeared to show a double-peaked profile with six Gaussian emission components, having originated in the accretion disk which consists of two or more ring structures. The center wavelengths of the broad emission Gaussian components of $\mathrm{H} \gamma$ and $\mathrm{H} \delta$ were almost in agreement with the wavelength $\lambda_{0}$ corresponding the system velocity. However, those of $\mathrm{H} \alpha$ and $\mathrm{H} \beta$ were a little asymmetric with respect to $\lambda_{0}$. In this case, these asymmetric distributions of $\mathrm{H}$ I gases around the white dwarf could be considered. However, it is uncertain whether these asymmetries were due to a tidal effect by the third object or due to a highly eccentric orbit motion, where $i \approx 90^{\circ}$. The double-peaked emission components of He I were also considered to have been formed in the inner region of the disk.

On the other side, Fe II and [Fe II] lines with single emission line components were considered to have originated by means of radiative ionization and recombination processes close to the white dwarf.

Triple peaked emission components of $\mathrm{He}$ II and [O III] might be formed at two adjacent regions to each other. Emission components of He II and [O III] had 
TABLE 2.

Parameters of the Gaussian components for [O III], [Fe VI] And [Fe VII].

\begin{tabular}{|c|c|c|c|c|c|c|c|}
\hline $\begin{array}{l}\text { Date } \\
2004 \\
\end{array}$ & $\begin{array}{c}\text { wavelength } \\
(\AA)\end{array}$ & Component & $\overline{\lambda_{0}}$ & $\overline{\mathrm{I}}$ & $\begin{array}{c}\text { FWHM } \\
(\AA)\end{array}$ & $\begin{array}{r}V_{r} \\
\left(\mathrm{~km} \mathrm{~s}^{-1}\right) \\
\end{array}$ & $\begin{array}{r}V_{D} \\
\left(\mathrm{~km} \mathrm{~s}^{-1}\right) \\
\end{array}$ \\
\hline \multirow[t]{6}{*}{ April 9} & & (1) & 4957.03 & 2.50 & 0.27 & -113.6 & 19.7 \\
\hline & [O III] & (2) & 4957.64 & 24.10 & 0.65 & -77.7 & 47.4 \\
\hline & 4958.91 & $(3)$ & 4958.78 & 3.10 & 0.84 & -7.9 & 61.2 \\
\hline & & $\mathrm{B}$ & 4958.98 & 4.10 & 3.44 & +4.3 & 250.7 \\
\hline & & (4) & 4959.23 & 6.80 & 0.48 & +19.6 & 35.0 \\
\hline & & $(5)$ & 4960.04 & 7.80 & 0.82 & +68.1 & 59.8 \\
\hline \multirow[t]{6}{*}{ April 9} & & (1) & 5005.00 & 7.50 & 0.29 & -110.2 & 20.9 \\
\hline & [O III] & (2) & 5005.58 & 58.90 & 0.56 & -75.2 & 40.4 \\
\hline & 5006.84 & (3) & 5006.46 & 5.00 & 0.78 & -23.0 & 56.3 \\
\hline & & $\mathrm{B}$ & 5006.98 & 11.00 & 2.85 & +8.5 & 205.7 \\
\hline & & (4) & 5007.14 & 11.80 & 0.47 & +17.9 & 33.9 \\
\hline & & $(5)$ & 5008.00 & 18.30 & 0.70 & +69.7 & 50.5 \\
\hline \multirow[t]{5}{*}{ April 9} & & (1) & 5174.84 & 11.30 & 0.79 & -83.1 & 55.2 \\
\hline & {$[\mathrm{Fe} \mathrm{VI}]$} & (2) & 5175.94 & 2.00 & 0.42 & -19.2 & 29.3 \\
\hline & 5176.43 & $\mathrm{~B}$ & 5176.45 & 3.00 & 2.85 & +10.3 & 199.0 \\
\hline & & $(3)$ & 5176.77 & 2.00 & 0.47 & +28.9 & 32.8 \\
\hline & & (4) & 5177.78 & 3.70 & 0.68 & +87.5 & 37.5 \\
\hline \multirow[t]{5}{*}{ April 9} & & (1) & 5719.53 & 6.20 & 1.05 & -71.8 & 68.4 \\
\hline & {$[\mathrm{Fe} \mathrm{VII}]$} & (2) & 5720.67 & 2.00 & 0.41 & -12.0 & 25.9 \\
\hline & 5720.9 & $\mathrm{~B}$ & 5720.88 & 5.30 & 2.73 & -1.35 & 172.5 \\
\hline & & $(3)$ & 5721.60 & 3.60 & 0.60 & +36.6 & 37.9 \\
\hline & & (4) & 5722.63 & 8.50 & 0.79 & +90.6 & 49.9 \\
\hline
\end{tabular}

B: Gaussian broad emission component.

$(1),(2) \ldots(5)$ : Gaussian emission components in the order of wavelength.

TABLE 3.

RAdial VElocities of PERMitTed Lines $\left(\mathrm{km} \mathrm{s}^{-1}\right)$.

\begin{tabular}{c|c|c|c|c}
\hline \hline H I & He I & He II & \multicolumn{1}{c|}{ O I } & Fe II \\
\hline$-73.5 \pm 1.8$ & $-74.5 \pm 7.0$ & $-74.1 \pm 1.2$ & -72.1 & \\
& & & & \\
& $+13.7 \pm 9.0$ & & -8.4 & $+10.9 \pm 1.0$ \\
& & $+25.8 \pm 1.2$ & +30.6 & \\
$+56.1 \pm 3.8$ & $+61.6 \pm 5.6$ & & & \\
$+82.0 \pm 3.4$ & & $+79.2 \pm 2.4$ & +89.3 & \\
\hline
\end{tabular}

1. The $\mathrm{H}$ I lines are $\mathrm{H} \alpha, \mathrm{H} \beta$ and $\mathrm{H} \gamma$.

2. The He I lines are $4471 \AA, 4922 \AA, 5016 \AA, 5876 \AA, 6678 \AA, 7065 \AA$ and $7281 \AA$.

3. The He II lines are $4686 \AA$ and $5412 \AA$.

4. The O I line is $7156 \AA$.

5. The Fe II lines are $5018 \AA, 5198 \AA, 5317 \AA$ and $5425 \AA$.

much identical radial velocities. However different densities of the regions producing He II and [O III] lines were anticipated. The densities of regions forming forbidden lines were well known to be lower.

N III was observed around $\lambda 4640$ as a nebular line before the second eclipse of 1993 (Andrillat \& Houzi- aux, 1995; Kolotilov et al., 1995). The shell structures might be evolved slowly for such complex line shapes. [Ar III] and [Fe VII] were observed as a single component during the second eclipse phase of 1993 with intensities smaller than those before the eclipse (Andrillat \& Houziaux, 1995). During the eclipse, the white dwarf was eclipsed by the cool M giant. However, in April 
TABLE 4.

RADIAL VELOCITIES OF FORBIDDEN LINES $\left(\mathrm{km} \mathrm{s}^{-1}\right)$.

\begin{tabular}{|c|c|c|c|c|c|c|c|c|c|c|}
\hline [N II] & {$\left[\begin{array}{ll}\mathrm{O} & \mathrm{I}\end{array}\right.$} & [O III $]$ & [Ne IV] & {$[\mathrm{Ca} \mathrm{V}]$} & [Ar III] & [Ar IV] & {$[\mathrm{Ar} \mathrm{V}]$} & {$[$ Fe II] } & {$[\mathrm{Fe} \mathrm{VI}]$} & {$[\mathrm{Fe} \mathrm{VII}]$} \\
\hline & \multirow{5}{*}{$+9.0 \pm 4.0$} & & $-115.0 \pm 14.2$ & & & & & \multirow{5}{*}{$10.9 \pm 6.3$} & -104.2 & \\
\hline-67.9 & & $-66.9 \pm 0.5$ & $-31.5 \pm 6.3$ & -61.4 & -69.8 & $\begin{array}{l}-74.0 \\
-47.3\end{array}$ & -77.7 & & -58.4 & -57.4 \\
\hline \multirow{3}{*}{$\begin{array}{l}16.5 \\
55.1\end{array}$} & & \multirow{3}{*}{$\begin{array}{l}25.2 \pm 3.0 \\
74.7 \pm 3.6\end{array}$} & \multirow{3}{*}{$34.3 \pm 0.3$} & 0.2 & -12.6 & & \multirow{3}{*}{51.6} & & -1.4 & $-2.9 \pm 1.1$ \\
\hline & & & & 48.8 & \multirow[b]{2}{*}{65.6} & & & & & $49.0 \pm 3.1$ \\
\hline & & & & 103.0 & & & & & 65.2 & $134.8 \pm 24.6$ \\
\hline
\end{tabular}

1. The [N II] line is $5755 \AA$.

2. The $[\mathrm{O}$ I] lines are $6300 \AA$ and $6364 \AA$.

3. The [O III] lines are $4363 \AA, 4959 \AA$ and $5007 \AA$.

4. The [Ne IV] lines are $4715 \AA$ and $4725 \AA$.

5. The $[\mathrm{Ca} \mathrm{V}]$ line is $5309 \AA$.

6. The [Ar III] line is $7136 \AA$.

7. The $[\mathrm{Ar} I V]$ line is $7171 \AA$.

8. The $[\mathrm{Ar} \mathrm{V}]$ line is $7006 \AA$.

9. The [Fe II] lines are $4966 \AA, 5262 \AA$ and $5334 \AA$.

10. The $[\mathrm{Fe} \mathrm{VI}]$ line is $5632 \AA$.

11. The [Fe VII] lines are $5721 \AA$ and $6086 \AA$.

2004, they were observed as nebular line profiles. The nebular lines were stronger in April 2004 than in 1993 and they were interpreted by the wind-shock model. Highly ionized elements of [Fe VI], [Fe VII], [Ca V] and [Ne IV] showed nebular lines in April 2004 which could also be explained by the same scenario.

All of expanding matter seemed to have symmetric shell structures with symmetric average velocities that were the grouped representatives of emission components of forbidden lines in Table 4. If the emission lines were superposed on flat-top profile as blends, they would have originated from blob-like cloudlets of the symmetric shell structures, which were observed as line complexes in April 2004.

The circumstellar space might have been continuously filled in the differentiated wind forms due to collisions between interstellar matter and material ejected from the white dwarf. In April 2004, evolutions in line intensities and features might have become noteworthy in terms of their origins. PU Vul seems to have experienced sudden flare around the end part of 2006(AAVSO), even though the brightness of PU Vul appears to go on declining. Thus, the investigation of long-spread-out developments of PU Vul are necessary with further observations.

\section{ACKNOWLEDGEMENTS}

The author would be grateful to Professor Yamashita for his helpful comments of improving this work and would like to thanks to the referee for some helpful comments. We acknowledge with thanks the variable star observations from the AAVSO International Database contributed by observers worldwide and used in this research.

\section{REFERENCES}

Andrillat, Y., Ciatti, F. Y., \& Swings, J. P., 1982, Visible and/or near infrared spectral variations of V1016 Cygni, HM Sagittae and MWC 349, Ap\&SS, 83, 423

Andrillat, Y. \& Houziaux, L., 1994, Spectroscopic observations of $\mathrm{Pu}$-Vulpeculae in the 3210 to $10950 \mathrm{~A}$ range during the nebular phase, MNRAS, 271, 875

Andrillat, Y. \& Houziaux, L., 1995, PU Vul during the Brightness Weakening of 1993-1994, IBVS, 4251, 1

Belyakina, T. S., Bondar, N. I., Chochol, D., Chuvaev, K. K., Efimov, Y. S., Gershberg, R. E., Grygar, J., Ilric, L., Krasnobabtshev, V. I., Petrov, P. P., Piirola, V., Savanov, I. S., Shakhovskaya, N. I., Shakhovskoj, N. M., \& Shenavrin, V. I., 1984, 
The Kuwano-Honda peculiar object (PU Vulpeculae), A\&A, 132, 12

Belyakina, T. S., Bondar, N. I., Chochol, D., Chuvaev, K. K., Efimov, Y. S., Gershberg, R. E., Grygar, J., Hric, L., Krasnobatsev, V. I., Piirola, V., Poutanen, M., Savanov, I. S., Huovelin, J., Tuominen, I., Shakhovskaya, N. I., Shakhovskoy, N. M., Shenavrin, V. I., \& Scherbakov, A. G., 1989, The Kuwano-Honda's peculiar object (PU Vulpeculae) in 1983-1986, A\&A, 223, 119

Belyakina, T. S., Gershberg, R. E., Efimov, Y. S., Krasnobabtsev, V. I., Pavlenko, E. P., Petrov, P. P., Chuvaev, K. K., \& Shenavrin, V. I., 1982, Kuwano's peculiar object - A nova like symbiotic binary system containing a red giant - Discussion of observational results, SvA, 26, 184

Bensammar, S., Chauville, J., Letourneur, N., \& Friedjung, M., 1991, The infrared spectrum of the eruptive star PU Vulpeculae, A\&A, 245, 575

Chochol, D., Pribulla, T., \& Tamura, S., 1998, Pulsating AGB star in the symbiotic nova PU Vulpeculae, IBVS, 4571, 1

Chochol, D., Pribulla, T., Tamura, S., Tajitsu, A., \& Kanamitsu, O., 1997, Physical processes in the very slow symbiotic nova PU Vul - Possible triple system, PPSB. Conf., 127

Chochol, D., Pribulla, T., Parimucha, S., \& Vanko, M., 2003, Long-term photometry of very slow novae, BalA, 12, 610

Fitzgerald, M. P., \& Pilavaki, A., 1974, V1016 Cygni: Spectral observations 1969-1973, ApJS, 28, 147

Gochermann, J., 1991, PU Vulpeculae at the time of spectral change in 1987, A\&A, 250, 361

Hoard, D. W., Wallerstein, G., \& Willson, L. A., 1996, ROSAT Observations of the symbiotic stars PU Vulpeculae and FG Serpentis, and the cataclysmic variable V Sagittae, PASP, 108, 81

Honda, M., Ishida, K., Noguchi, T., Norimoto, Y., Nakagiri, M., Soyano, T. \& Yamashita, Y., 1979, Observations of a novalike object in Vulpecula, TokAB, 262,2983

Iben, I. Jr., \& Tutukov, A. V., 1996, On the evolution of symbiotic stars and other binaries with accreting degenerate dwarfs, ApJS, 105, 145

Iijima, T., 1989, Recent spectral variation of the peculiar nova-like object PU Vulpeculae, A\&A, 215, 57

Iijima, T., \& Ortolani, S., 1984, Spectral variation and the expanding envelope of PU Vulpeculae, A\&A, 136,1

Ikeda, Y., \& Tamura, S. I., 2004, Spectroscopic diagnostics of symbiotic stars. II. Statistical analyses of highly resolved emission-line profiles, PASJ, 56, 353

Kanamitsu, O., 1991, A spectroscopic study of PU Vulpeculae, PASJ, 43, 225
Kanamitsu, O., Yamashita, Y., Norimoto, Y., Watanabe, E., \& Yutani, M., 1991, Weak-line to emissionline phases of PU Vulpeculae, PASJ, 43, 523

Kenyon, S. J., 1986, Spectroscopic observations of PU Vulpeculae, AJ, 91, 563

Kim, K. M., 2004, PhD thesis

Klein, A., Bruch, A., \& Luthardt, R. 1994, The development of the symbiotic nova PU Vulpeculae in the years 1984-1992, A\&AS, 104, 99

Kolotilov, E. A., 1989, Optical Variability of $\mathrm{Pu}-$ Vulpeculae - Kuwano's nova like object of 1979 The start of a fading stage, SvAL, 15, 223

Kolotilov, E. A., Munari, U., \& Yudin, B. F., 1995, On the new minimum of the symbiotic nova PU Vulpeculae, MNRAS, 275, 185

Kuwano, Y., 1979, Novalike object in Vulpecula, IAUC, Nos. 3344,3348

Liller, H. M., \& Liller, W., 1979, The pre-maximum light curve of the slow nova Vulpeculae 1979, AJ, 84, 1357

Maitzen, H. M., Schnell, A., \& Hron, J., 1987, PU Vulpeculae, IAUC, 4474, 2

Moore, C. E., 1958, A mutliplet table of astrophysical interest, national bureau of standards, technical note, No. 36

Nakagiri, M., \& Yamashita, Y., 1980, Photometric observations of Honda-Kuwano object in Vulpecula, TokAB, 263, 2993

Netopil, M., 2002, A quarter century PU Vulpeculae, HvaOB, 26, 37

Nussbaumer, H., \& Vogel, M., 1996, PU Vulpeculae: an eclipsing symbiotic nova, A\&A, 307, 470

Tamura, S. I., Kanamitsu, O., \& Yamashita, Y., 1992, Abrupt change of the $\mathrm{H}$-alpha emission-line profile of PU Vulpeculae in 1989, PASJ, 44, 543

Thackeray, A. D., \& Webster, B. L., 1974, The evolution of the Wolf-Rayet features of the slow nova, RR Telescopii, MNRAS, 168, 101

Tomov, T., Zamanov, R., Iliev, L., Mikolajewski, M., \& Georgiev, L., 1991, Wolf-Rayet features observed in the spectrum of the symbiotic nova PU Vulpeculae, MNRAS, 252, 31

Vogel, M. \& Nussbaumer, H., 1992, PU Vulpeculae The outburst of a symbiotic nova, A\&A, 259, 525

Yamashita, Y., Maehara, H., \& Norimoto, Y., 1982, Spectroscopic Observations of Pu-Vulpeculae / Nova Vulpeculae 1979, PASJ, 34, 269

Yamashita, Y., Norimoto, Y., \& Yoo, K. H., 1983, Spectral change of PU Vulpeculae (Nova VUL 1979), PASJ, 35, 521

Yamashita, Y., 1979, Observations of a novalike object in Vulpecula, TokAB, 262, 2983

Yoo, K. H., 2005, The high resolution spectra of PU Vul in 2004, PKAS, 21, 35 